\title{
The Effective QR Code Technology Development
}

\author{
Biyu Yuan ${ }^{1, a}$, Xiuping Zhao ${ }^{2, *}$ and Dapeng $\mathrm{Cui}^{2}$ \\ ${ }^{1}$ College of Packaging and Printing Engineering ,Tianjin University of Science \& Technology, \\ Tianjin 300222, china; \\ ${ }^{2}$ College of Packaging and Printing Engineering ,Tianjin University of Science \& Technology, \\ Tianjin 300222, china; \\ ${ }^{3}$ College of Packaging and Printing Engineering ,Tianjin University of Science \& Technology, \\ Tianjin 300222, china;

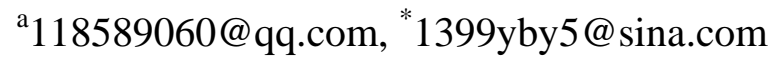

Keywords: QR Code, Security, Internet of Things, Trend.

\begin{abstract}
Based on the development trend of internet+ and the popularization of mobile intelligent terminals, anti-counterfeiting based on two-dimension code is also paid more and more attention by people. How to achieve higher anti-counterfeiting function through two-dimension code and realize product traceability and data statistics under the era of big data is one of the hot spots for people's research. How to prevent the counterfeit of two-dimensional code is the key point of Internet of things, Internet+ era under the hot spots of people's anti-counterfeiting research, this paper introduces the development of two-dimensional code technology and popular QR code technology which is popular and efficient nowadays.
\end{abstract}

\section{Similarity and Difference of Mainstream Two-dimensional Code Recognition}

QR Code is a type of 2D ${ }^{[1]}$ barcode that was invented and started to be used by Japanese Toyota subsidiary Denso Wave in 1994. The QR code can store more information than an ordinary barcode, nor does it need to be aligned like a normal barcode with the scanner in a straight line ${ }^{[2]}$.

Two-dimensional code technology can be divided into two kinds of layered and matrix. Identification Mechanism ${ }^{[2,3]}$ : Cascading is identified by the width of the black-and-white lines. The matrix is identified by the relative color difference of the black-and-white color blocks. The dark color module represents " 1 " in binary, and the light color module represents " 0 " in binary. So the general matrix is used to ensure that the color of a certain color.

Reading range: stacked identification equipment and bar code recognition angle difference of \pm $10^{\circ}$ or so, the basic matrix can achieve $360^{\circ}$ scan recognition.

Reading speed: One of the fastest QR code, which basically dozens of each second, and Data Matrix code and PDF417 basic speed of several per second.

\section{Effective QR Code Technology}

QR code involves a variety of technologies: physical anti-counterfeiting technology, traceability technology, digital technology, holographic technology. The most prominent feature is the dependence on special equipment is relatively small, and the relative low cost, which is now second dimensional code is still one of the main reasons for the market share, and the current QR code is largely open source algorithm, it is easy to be copied.

At present, the two-dimensional code technology that can achieve security is basically a combination anti-counterfeiting technology, which can be summarized as the following two-dimensional code and security code ${ }^{[2]}$, a combination of special ink and two-dimension code, a combination of specific holography technology and two- Technology and two-dimensional code combination, special APP and two-dimensional code technology, watermarking technology and two-dimensional bar code technology, invisible two-dimensional code technology. 


\subsection{Two-dimensional Code+ Security Code}

The two-dimensional code is printed on the commodity, the digital verification code is printed on the two-dimensional code, and the coating is covered by the coating. The digital verification code needs to be scratched for verification, the digital verification code and the two-dimensional code are related to the database, Dimensional code to save the product information, and in the box there is a box code associated with the two-dimensional code, box code and dealer associated, so that two-dimensional code can be extracted in the background database to the relevant products, distributors And other information ${ }^{[4]}$. only when the two-dimensional code A and security code B at the same time corresponding to prove their authenticity, if the current query is not the first query, indicating that the label may have been previously checked, anti-counterfeit code was scratched off, is a fake product.

\subsection{Specific Ink and Two-dimensional Code Combination technology}

Through the specific infrared or UV inks, the invisible pattern can be detected under the excitation of an external laser by using the principle that the red (purple) ink is invisible under visible light ${ }^{[2,4]}$. The two-dimensional code is identified by the color difference between the colors, so the light irradiation in a special light source will show its hidden patterns, identified by specific equipment, the technology can be achieved by 2 ways, one is the ink Printing in the entire pattern, one is the first printing of red (purple) outside the ink, and then printed on the ink to cover the other inks, and then scanned by the device to identify. Such anti-counterfeit ink is made of one or several near-red (purple) absorbing materials added to the ink.

\subsection{Specific Holographic Technology and Two-dimensional Code Combination}

This method is to laser holographic high-cost equipment, the two-dimensional code pattern hidden in this laser holographic image, only through a specific amplification equipment to identify, this technology is characterized by high cost, hidden more hidden, but The basic flow is not suitable for general use ${ }^{[4,5,6]}$. But the advantage lies in the technical difficulty and higher production costs, income and expenditure that are not equal and counterfeiters who are reluctant to counterfeit.

\subsection{Specific Technology and Two-dimensional Code Combination}

The two-dimensional code encryption is realized by a specific method. In combination with a dedicated APP recognition and a nano two-dimensional code anti-counterfeiting technology, a variable two-dimensional code template is mainly reserved for a variable data area, and a variable data area is loaded with a product ID ${ }^{[4,5]}$. The technical features of the nano-plate are hidden in the nano-level numerous points, and these points is the fake production process, the number, location unattainable. The product information through the late inkjet sprays in the reserved area. And then through they own APP scan code to achieve. Each of its two-dimensional code test has the number of times, place records ${ }^{[6]}$. After a certain number of times the code automatically scrapped, no longer have test. It can realize the requirement of one object and one code, and can also realize one type of code while considering the cost, that is, do not reserve the variable data area and perform only one type of code recognition.

\subsection{Dedicated APP and Two-dimensional Code Technology}

Special APP through a specific code combined with a specific scanning APP to achieve anti-counterfeiting technology, most of its encryption methods can be divided into two types, symmetric encryption and asymmetric encryption algorithm, which are basically plaintext (unprocessed raw data) ${ }^{[1,5,7]}$. The secret key is encrypted, and the key is decrypted in the encrypted ciphertext (the new data information that is different from the original data and encrypted by the algorithm). The principle is shown in figure 1, where the secret key 1 and the secret key 2 are the same which is called symmetric encryption. Secret keys 1 and 2 are called asymmetric encryption. Among them, symmetric encryption algorithms mainly include DES algorithm, DES algorithm, TDEA algorithm, Blowfish algorithm, RCS algorithm and IDEA algorithm. Asymmetric encryption algorithms RSA, Elgamal, backpack algorithm, Rabin, HD, elliptic curve encryption algorithm (ECC algorithm). The implementation of DES algorithm mainly combines three kinds of cryptography such as permutation, displacement and replacement, and adopts an iterative algorithm structure. The process of DES encryption of the original to-be-encrypted information is generally 
divided into three stages: the initial packet bit position replacement, multiple rounds of substitutions and substitutions, and permutations of group bits. RSA algorithm is based on the analysis of some mathematical problems based on the scientists summed up, and its realization depends on two important theoretical and mathematical foundations: Reversible modulus exponent and Eider Theorem (Euler's theorem), the amount of computation and generated secret keys are unpredictable so to some extent, reached a security. Currently available on the market for commodity-specific encryption algorithms generally use asymmetric encryption, which can meet the security requirements. The difference between the two algorithms lies in that the DES algorithm belongs to the single-key encryption system and the RSA algorithm belongs to the public-key encryption system. The former is symmetric encryption and the latter is asymmetric. The security RSA algorithm is far better than the DES algorithm. However, The overall difficulty of the DES algorithm is better ${ }^{[8]}$.

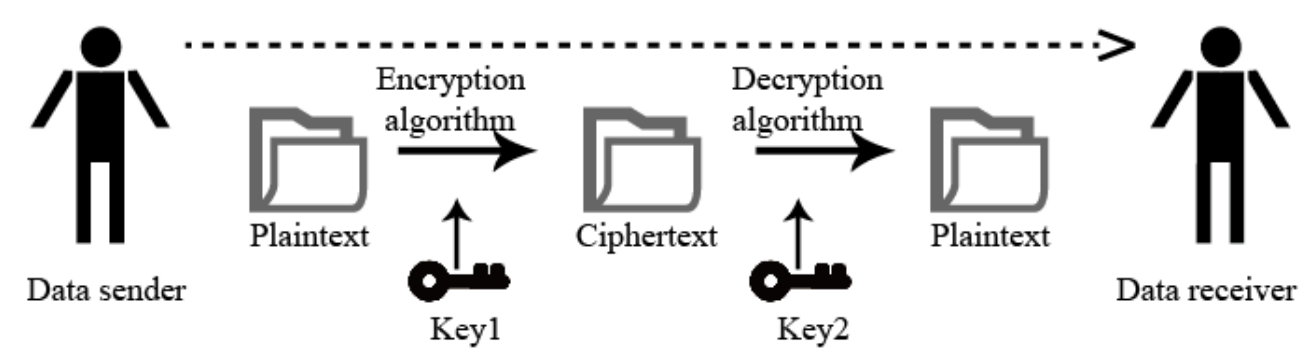

Fig. 1 Data encryption process

\subsection{Watermarking Technology and Two-dimensional Bar Code Technology}

Watermark embedding algorithm, we must first QR code images and watermarking images were processed, QR code images should be discrete wavelet transform and singular value decomposition, watermarking images should be first chaotic encryption, embedding watermarking after singular value decomposition and anti-discrete Wavelet changes, and finally get the required watermark bar code $^{[9,10]}$.

To ensure the robustness of the watermark, the embedding intensity of the watermark must be large enough, but the visual quality of the image should not be damaged. Therefore, an appropriate watermark embedding intensity factor should be chosen. Within a certain range, as the embedding factor shrinks, the watermark extracted will gradually become clearer. When an optimal value is reached, the reduction of the embedding factor will be to extract the watermark to become blurred again. Therefore, the embedded watermark intensity factor is the core technology of watermarking. Its characteristic is that the transformation of watermark encryption is relatively confidential and embedded watermark (pattern) clarity due to different intensity factors there is a big difference, through the naked eye observation.

\section{Conclusion}

How to create a more convenient anti-counterfeiting technology with stronger anti-counterfeiting technology and relatively lower cost is the goal that anti-counterfeiting technology and computer field have been exploring. With the development of computer network and the popularization of intelligent terminals, how to combine the current social environment and how seize this opportunity are also a challenge for anti-counterfeit enterprises and technical individuals. The future of the two-dimensional code security is bound to be able to do both the one thing and one product traceability and anti-channel conflict, and can be cheaper and more convenient direction. 


\section{References}

[1] A.M. Martinez-Grana, J.L. Goy, C.A. Cimarra. A virtual tour of geological heritage: valourising geodiversity using Google earth and QR code, J. Computers \& Geosciences. 61 (2013) 83-93.

[2] M. Meng, J.H. Sun, Z.S Deng. System design and realization of tropical fruit quality safety traceability based on 2D bar code Technology, J. Packaging Engineering. 275 (2014) 13-20.

[3] Y.H. Zhao. Application of RFID technology in the anti-counterfeit packaging, J. Printing Quality \& Standardization. 183 (2013) 5-19.

[4] L.X. Zhang, X.H. Tao et al. Application of QR code in the banknote anti-counterfeit technology, J. Development \& Innovation of Machinery \& Electrical Products. 132 (2012) 25-27.

[5] Y. Wei, A.M. Yan, J.B. Dong. Optical image encryption using QR code and multilevel fingerprints in gyrator transform domains, J. Optics Communications. 403 (2017) 62-67.

[6] P.P. Thulasidharan, M.S. Nair. QR Code based blind digital image watermarking with attack detection code, J. Aeu-international Journal of Electronics and Communications. 69 (2015) 1074-1084.

[7] Y.Peng, B.T. Xiao. Research and application of digital anti-counterfeit, J. Software Guide. 81(2009) 144-145.

[8] T. Green, T. Smith, R. Hodges et al. A simple and inexpensive way to document simple husbandry in animal care facilities using QR code scanning, J. Laboratory Animals. 51 (2017) 656-659.

[9] X.T. Wang, J.P. Qian, J.Li. A real-time agro-food authentication and supervision system on a novel code for improving traceability credibility, J. Food Control. 66 (2016) 12-26.

[10] L. Li, J.X. Qiu, J.F. Lu. An aesthetic QR code solution based on error correction mechanism, J. Journal of Systems and Software. 116 (2016) 85-94. 